\title{
Análisis del gasto en salud reproductiva en México, 2003
}

\author{
Lucero Cahuana-Hurtado, ${ }^{1}$ Leticia Ávila-Burgos, ${ }^{1}$ \\ Ricardo Pérez-Núñez y Patricia Uribe-Zúñiga ${ }^{2}$
}

Forma de citar Cahuana-Hurtado L, Ávila-Burgos L, Pérez-Núñez R, Uribe-Zúñiga P. Análisis del gasto en salud reproductiva en México, 2003. Rev Panam Salud Publica. 2006;20(5):287-98.

RESUMEN Objetivos. Estimar el gasto en salud reproductiva en México durante el año 2003, analizar su distribución según los principales programas, agentes de financiamiento y proveedores de bienes y servicios de salud, y evaluar la relación entre el gasto en salud reproductiva y algunos indicadores económicos de los estados, mediante la metodología de cuentas en salud.

Métodos. Se estimó el gasto en salud reproductiva entre enero y diciembre de 2003, tanto a nivel nacional como estatal. Se utilizó la metodología de cuentas en salud ajustada a las particularidades de México a partir de información pública y privada. El gasto se calculó para los cuatro principales programas de salud reproductiva (salud materno-perinatal, planificación familiar, cáncer cervicouterino y cáncer de mama) según los diferentes agentes de financiamiento, proveedores de bienes y servicios y funciones de salud, tanto para el sector público como privado. Se estimó el gasto público estatal por beneficiaria y se analizó su relación con el gasto público en salud y el producto interno bruto (PIB) anual per cápita de cada estado.

Resultados. El gasto en salud reproductiva en México durante el año 2003 fue de 2 912,6 millones de dólares estadounidenses y representó 0,5\% del PIB nacional en 2003 y poco más de $8 \%$ del gasto en salud. El gasto fue mayor en los agentes públicos $(53,5 \%)$ que en los privados $(46,5 \%)$. El programa de salud materno-perinatal presentó el mayor gasto, principalmente por partos y complicaciones; casi $50 \%$ de ese total provino de pagos directos de los hogares. El gasto en planificación familiar fue mayormente público y representó 5,9\% del gasto total. Del gasto en salud reproductiva, 7,9\% correspondió a los programas de cáncer cervicouterino y de mama. El gasto público promedio en salud reproductiva por beneficiaria fue de 680,03 USD y su distribución estatal estuvo asociada con el gasto público en salud ( $\mathrm{r}=0,80$; $\mathrm{P}<0,001)$ y el PIB per cápita $(\mathrm{r}=0,75 ; \mathrm{P}<0,0001)$.

Conclusiones. La metodología de cuentas en salud permitió estimar el gasto en salud reproductiva en México en 2003. Fortalecer las acciones y los programas de salud reproductiva a partir de una asignación del gasto basada en la evidencia y enfocada a las poblaciones más desfavorecidas es un imperativo ético, de derechos humanos y de desarrollo.

Palabras clave Gastos en salud, servicios de salud reproductiva, México.

1 Instituto Nacional de Salud Pública, Centro de Investigación en Sistemas de Salud, Cuernavaca, Morelos, México. La correspondencia se debe dirigir a Leticia Ávila Burgos, Avenida Universidad No. 655, Colonia Santa María Ahuacatitlán, Cuernavaca, Morelos CP 62508, México. Correo electrónico: lavila@insp.mx

2 Secretaría de Salud, Centro Nacional de Equidad de Género y Salud Reproductiva, México, D. F., México.
Los procesos de descentralización de los servicios de salud en México otorgaron a los estados independencia para asignar sus recursos de acuerdo con las prioridades nacionales y locales de salud. Esto generó la necesidad de contar con la información finan- ciera que permitiera conocer el monto de los recursos asignados a las áreas prioritarias de salud. Para satisfacer esa necesidad se creó el Sistema de Cuentas en Salud a Nivel Federal y Estatal (SICUENTAS) $(1,2)$, que brinda información mediante una estructura 
que estandariza los gastos según las funciones generales del sistema de salud. Sin embargo, a pesar de lo detallado de esa información, su desglose no es suficiente para identificar el gasto realizado en actividades específicas y poder evaluar el desempeño del sistema de salud.

La falta de información específica en salud reproductiva imposibilita monitorear el impacto del proceso de descentralización en la asignación de recursos a actividades consideradas prioritarias en esta área. Además, no permite conocer si la distribución actual del gasto se adecua a la distribución geográfica de las necesidades de salud. Esta carencia de información cobra especial importancia si se tiene en cuenta que en 2003, 1313 mujeres fallecieron por trastornos obstétricos - como hipertensión durante el embarazo $(31 \%)$ y hemorragias durante el embarazo y el parto (26\%) - evitables con intervenciones poco costosas (3). La mayoría de estos fallecimientos ocurrieron en los estados más pobres de México. Debido a esta desigualdad, una mujer de un estado considerado pobre, como Guerrero, presentó ocho veces más probabilidades de morir por trastornos obstétricos que una mujer de un estado considerado rico, como Nuevo León (3). En relación con la salud perinatal, la Secretaría de Salud señaló que en el año 2003, dos de cada tres muertes infantiles ocurrieron antes de alcanzar la primera semana de vida y $20 \%$ del total fallecieron durante las primeras 24 horas después del nacimiento. Más de $70 \%$ de las muertes infantiles estuvieron asociadas con afecciones perinatales o malformaciones congénitas (4).

Los tipos de cáncer que más muertes provocan en mujeres mexicanas son el cervicouterino y el de mama. Similar a lo que sucede con la mortalidad materna, la prevalencia de cáncer cervicouterino ha sido mayor en las zonas rurales y en los estados más pobres, mientras que el cáncer de mama es más frecuente en los estados con mayor desarrollo (5).

En 2003, el sistema de salud mexicano estaba integrado por el sector privado - que abarcaba los servicios de salud provistos por instituciones particulares, las aseguradoras privadas y las organizaciones no gubernamentales que ofrecen atención a la salud-y el sector público, compuesto por la seguridad social y los servicios del gobierno orientados a la población de escasos recursos. De las cinco instituciones que integraban la seguridad social, las de mayor cobertura eran el Instituto Mexicano del Seguro Social (IMSS), que cubría más de $40 \%$ de la población $(6,7)$ y el Instituto de Seguridad y Servicios Sociales para los Trabajadores del Estado (ISSSTE), que atendía a los empleados del sector público (10\% de la población mexicana) $(7,8)$. Como parte del proceso de reforma del sector salud mexicano, ese año se introdujo en fase piloto el Seguro Popular de Salud, un seguro social voluntario destinado a la población sin acceso a otras formas de seguridad social.

Las principales instituciones del gobierno destinadas a atender a las personas de escasos recursos sin acceso a la seguridad social eran la Secretaría de Salud y los Servicios Estatales de Salud, así como el programa Oportunidades del IMSS (IMSS-Oportunidades). Se calcula que $24 \%$ de la población recurrió a este tipo de instituciones (7). Los servicios del sector privado -que se caracterizan por una baja regulación y una gran heterogeneidad en cuanto a la calidad y al costo - atendieron a poco más de 30\% de la población, según las estadísticas oficiales (7). Un grupo muy reducido y privilegiado - alrededor de $2 \%$ de la población total- estaba asegurado por alguna compañía privada de seguros.

Como se observa, el financiamiento del sistema de salud mexicano es una mezcla público-privada y se caracteriza por una fuerte presencia del gasto privado. A pesar del incremento experimentado por el gasto público en los últimos años, el gasto privado continúa siendo el principal agente de financiamiento y en 2003 , cerca de $90 \%$ de ese gasto provino de los pagos directos de los hogares (9). En el caso del gasto público, la seguridad social desembolsó dos tercios de ese gasto, proporción que fluctuó desde $35 \%$ (en el estado de
Chiapas) hasta 87\% (en Nuevo León) (10). Según SICUENTAS, cerca de $90 \%$ del gasto en salud de ese año se dedicó a la atención directa, alrededor de 3\% se destinó a servicios de prevención y a acciones de salud pública y el resto a actividades de rectoría, administración y formación de recursos, entre otras. Menos de la mitad del gasto en salud se realizó en los hospitales (9). En conjunto, en 2003 se gastó alrededor de $6,0 \%$ del producto interno bruto (PIB) anual de México en actividades destinadas a la salud $(10,11)$.

Los objetivos de este estudio fueron estimar el gasto en salud reproductiva en México durante el año 2003, analizar su distribución según los principales programas, agentes de financiamiento y proveedores de bienes y servicios de salud, y evaluar la relación entre el gasto en salud reproductiva y algunos indicadores económicos de los estados, mediante la metodología de cuentas en salud.

\section{MATERIALES Y MÉTODOS}

Se estimó el gasto en salud reproductiva, tanto a nivel nacional como estatal, entre enero y diciembre de 2003. El gasto en salud reproductiva se desglosó en los cuatro programas de salud reproductiva de mayor cobertura y más recursos en México (salud materno-perinatal, planificación familiar, cáncer cervicouterino y cáncer de mama) y según los agentes de financiamiento (públicos y privados), los proveedores de bienes y servicios y las funciones de salud. Para ello se utilizaron los datos del estudio sobre cuentas en salud reproductiva, realizado por el Instituto Nacional de Salud Pública de México en 2004 (12), con financiamiento de la Secretaría de Salud a través del Centro Nacional de Equidad de Género y Salud Reproductiva. La estimación se realizó mediante la metodología de cuentas en salud, propuesta por el Banco Mundial, la Organización Mundial de la Salud (OMS) y la Agencia de Estados Unidos para el Desarrollo Internacional (USAID) (13), ajustada para las particularidades de México (14). Esta 
metodología permite documentar el flujo de fondos para la producción y el consumo de bienes y servicios a través del sistema de salud, mediante tablas de doble entrada (matrices).

\section{Definiciones operativas}

Según la definición de la OMS, utilizada por el Centro Nacional de Equidad de Género y Salud Reproductiva, la salud sexual y reproductiva comprende las estrategias dirigidas a contribuir a que las personas tengan una vida sexual, reproductiva y posreproductiva satisfactoria, saludable y sin riesgos, con respeto a sus derechos y a su libre decisión $(15,16)$. No obstante, en este trabajo la definición de salud reproductiva se restringió al conjunto de actividades relacionadas con cuatro programas específicos: a) salud materno-perinatal, que comprende la atención del embarazo, el parto y el puerperio, el aborto y la atención del recién nacido y sus complicaciones; b) planificación familiar, que comprende la provisión y el seguimiento de métodos anticonceptivos definitivos y temporales y la fertilización asistida; c) cáncer cervicouterino, que comprende la detección oportuna de este tipo de cáncer, las campañas de tamizaje y la atención de pacientes con cáncer o displasias; y d) cáncer de mama, que comprende la exploración mamaria, las campañas de tamizaje y la atención de pacientes con cáncer de mama o displasias. La definición de trabajo abarcó, además, las actividades de promoción de la salud, capacitación de personal, rectoría y administración y formación bruta de capital, así como las investigaciones relacionadas con la salud reproductiva (12). No se tomaron en cuenta las acciones dirigidas a los adolescentes, la atención de la menopausia y el climaterio y la atención del cáncer de próstata, como tampoco las dirigidas a la prevención y atención de las infecciones de transmisión sexual, ya que están comprendidas en las cuentas nacionales dedicadas a la infección por el virus de la inmunodeficiencia humana y el sida (17). Para una mayor homogeneidad, en el caso de la atención hospitalaria se uti- lizó la 10. a edición de la Clasificación Internacional de Enfermedades ${ }^{3}$ (18).

El gasto en salud reproductiva se definió como el monto de los recursos utilizados en las actividades comprendidas bajo la definición de trabajo de salud reproductiva. Su estimación se realizó a nivel estatal y solo se tomaron en cuenta las transacciones realizadas dentro del país entre enero y diciembre de 2003 (13). Se utilizó la información suministrada por la Secretaría de Salud, los Servicios Estatales de Salud, el IMSS, el ISSSTE y el programa IMSS-Oportunidades, así como la información sobre los pagos directos realizados por los hogares para la compra de servicios de salud reproductiva, los montos ejercidos por las aseguradoras privadas y por las dos organizaciones no gubernamentales (ONG) más grandes que trabajan en salud reproductiva: la Fundación Mexicana para la Planificación Familiar (MEXFAM) y la Federación Mexicana de Asociaciones Privadas de Planificación Familiar (FEMAP).

Para el cálculo del gasto se utilizaron las siguientes categorías de análisis (12-14):

Agentes de financiamiento: entidades que acumulan fondos y los asignan para adquirir bienes y servicios entre los proveedores del sector salud. Se consideraron cinco tipos de agentes: gobierno (Secretaría de Salud federal, Servicios Estatales de Salud e IMSSOportunidades), seguridad social (IMSS e ISSSTE), pagos directos de los hogares, empresas privadas de seguros y ONG. Estas categorías se agruparon en sector público (gobierno y seguridad social) y sector privado (pagos directos de los hogares, empresas privadas de seguros y ONG).

Proveedores: instituciones que reciben dinero para ofrecer bienes y servicios de salud. Se agruparon en: hospitales, proveedores de atención ambulatoria (tanto los consultorios médicos como

\footnotetext{
3 Los diagnósticos por programa se agruparon del siguiente modo: salud materno-perinatal: O99O92, P00-P96 y Z39; planificación familiar: N46.X, N97-N98, Z30-Z31; cáncer cervicouterino: C65, D06 y N87; y cáncer de mama: C50 y D05.
}

de otros profesionales de la salud, laboratorios y servicios de medicina tradicional y alternativa), farmacias, programas de salud pública (suministro, rectoría y administración) y servicios de administración general de salud.

Funciones de salud: actividades desempeñadas por instituciones o personas con el objetivo de mejorar el estado de salud de la población. Según el esquema utilizado por SICUENTAS en México (14), el gasto se agrupó en:

1. servicios de atención curativa, ambulatoria y hospitalaria

2. bienes proporcionados a pacientes ambulatorias, tales como los medios anticonceptivos y los suplementos vitamínicos durante el embarazo

3. servicios de prevención y de salud pública, entre ellos las consultas prenatales, de puerperio, de recién nacido y de planificación familiar, así como las campañas de promoción sobre salud reproductiva

4. rectoría y administración de la salud y los seguros médicos

5. formación de capital y de personal sanitario, de investigación y de desarrollo.

La categoría fuentes de financiamiento no se investigó por la dificultad de realizar el seguimiento de los fondos financieros hasta esa dimensión.

\section{Estimación del gasto}

Se siguieron dos estrategias para la estimación:

a) Mediante el ejercicio presupuestal, que consistió en la ponderación del gasto realizado con indicadores de los servicios otorgados. Este método se empleó para estimar el gasto de la seguridad social y de la Secretaría de Salud según la fórmula:

$\mathrm{G}_{i j}=\mathrm{P}_{i j}$ * $\left(\mathrm{Ssr}_{i j} / \mathrm{Ss}_{i j}\right)$ donde, $\mathrm{G}_{i j}$ es el gasto en salud reproductiva en la institución $i$ en el estado $j$; $\mathrm{P}_{i j}$ es el presupuesto ejercido para la atención ambulatoria $\mathrm{u}$ hospitalaria en la institución $i$ en el estado $j$; 
$\mathrm{Ssr}_{i j}$ es el indicador de los servicios de salud reproductiva prestados (por ejemplo, las consultas o los días de estancia hospitalaria por diagnóstico) en la institución $i$ en el estado $j ; \mathrm{y}$

$\mathrm{Ss}_{i j}$ es el total de los servicios prestados (por ejemplo, el total de consultas o el total de días de estancia hospitalaria) en la institución $i$ en el estado $j$.

b) Mediante los costos, calculado como el producto de los servicios otorgados por el costo promedio de cada actividad, según los datos publicados (19-23) y los documentos internos de la Secretaría de Salud. Esta metodología se utilizó para estimar el gasto del programa IMSSOportunidades y de las ONG.

$\mathrm{G}=\sum \mathrm{Ssr}^{*} \mathrm{C}$

donde,

$\mathrm{G}$ es el gasto en salud reproductiva; Ssr son los servicios de salud reproductiva prestados; y

C es el costo de los servicios de salud reproductiva.

Se solicitaron las bases de egresos hospitalarios y de consulta externa a cada una de las instituciones participantes en el estudio. Para utilizar las mismas categorías presupuestales entre instituciones y evitar la duplicidad, en la información de los presupuestos utilizados se especificaron los rubros contenidos en cada categoría de gasto.

Para estimar el gasto del sector privado se utilizaron los datos de las encuestas de hogares. El gasto monetario y no monetario empleado por los hogares para la atención del embarazo y del parto, así como para comprar métodos anticonceptivos, se tomó de la Encuesta Nacional de Ingreso y Gasto de los Hogares 2002 (ENIGH 2002) (24). ${ }^{4}$ Asimismo, se utilizaron los datos del uso de los servicios dedicados al diagnóstico y el tratamiento de cáncer cervicouterino y de mama contenidos en la Encuesta Nacional de Salud Re-

\footnotetext{
4 En la ENIGH 2002 se informa el gasto en anticonceptivos sumado al de vitaminas y otros productos. Por ello, para identificar el gasto exclusivo en anticonceptivos se utilizaron los datos de la ENIGH 2004 (25), en la que ambos rubros se presentan por separado.
}

productiva de 2003 (ENSAR 2003) (26). También se recabó información sobre el gasto de las ONG MEXFAM y FEMAP, así como de la Asociación Mexicana de Instituciones de Seguros, que cubre la población con seguro médico privado. Esta información se obtuvo de SICUENTAS (9).

\section{Análisis de los datos}

Los datos nacionales se ordenaron en dos matrices de gastos. La primera contenía el gasto en salud reproductiva según el agente de financiamiento y la función de salud, mientras que en la segunda se ingresó la información del gasto estimado en salud reproductiva según el proveedor y la función de salud (13).

Solo se pudo desagregar la información del gasto público hasta el nivel estatal. Con el fin de hacer comparaciones válidas entre estados, se dividió el gasto estatal de cada programa entre su respectiva población beneficiaria. Con la sumatoria de estas razones se conformó el gasto público en salud reproductiva por beneficiaria en cada estado. Los grupos beneficiarios se definieron a partir de normas oficiales y programas de acción. El gasto público en salud reproductiva por beneficiaria a nivel estatal se calculó mediante la siguiente fórmula:

$\mathrm{Gb}_{j}=\left(\mathrm{G}_{\mathrm{SMP} j} / \mathrm{B}_{\mathrm{SMP} j}\right)+\left(\mathrm{G}_{\mathrm{PF} j} / \mathrm{B}_{\mathrm{PF} j}\right)+$ $\left(\mathrm{G}_{\mathrm{CACU} j} / \mathrm{B}_{\mathrm{CACU} j}\right)+\left(\mathrm{G}_{\mathrm{CAMA} j} / \mathrm{B}_{\mathrm{CAMA} j}\right)$ donde,

$\mathrm{Gb}_{j}$ es el gasto público en salud reproductiva por beneficiaria en el estado $j$; $\mathrm{G}_{\mathrm{SMP} j}$ es el gasto público en salud materno-perinatal en el estado $j$;

$\mathrm{G}_{\mathrm{PF} j}$ es el gasto público en planificación familiar en el estado $j$;

$\mathrm{G}_{\mathrm{CACU} j}$ es el gasto público en cáncer cervicouterino en el estado $j$;

$\mathrm{G}_{\mathrm{CAMAj}}$ es el gasto público en cáncer de mama en el estado $j$;

$\mathrm{B}_{\mathrm{SMP} j}$ es la población beneficiaria del gasto en salud materno-perinatal: mujeres susceptibles de quedar embarazadas $^{5}$ en el estado $j$ (27);

\footnotetext{
5 Mujeres susceptibles de quedar embarazadas = (Población total * tasa cruda de natalidad)/1 000.
}

$\mathrm{B}_{\mathrm{PF} j}$ es la población beneficiaria del gasto en planificación familiar: mujeres entre 15 y 49 años en el estado $j$ (28); y

$\mathrm{B}_{\mathrm{CACU} j}$ y $\mathrm{B}_{\mathrm{CAMA} j}$ son las poblaciones beneficiarias del gasto en cáncer cervicouterino y de mama (mujeres mayores de 25 años), respectivamente, en el estado $j(29,30)$.

Se evaluó la asociación entre el gasto público en salud reproductiva por beneficiaria a nivel estatal y el gasto público per cápita en salud (10), y el gasto público en salud reproductiva por beneficiaria y el PIB per cápita del estado (11) mediante medidas de tendencia central y el índice de correlación de Spearman $(r)$.

Toda la información se expresa en dólares estadounidenses según el promedio de las tasas de cambio publicadas por el Banco de México durante el período de estudio (31). La información de las bases de datos se procesó mediante Stata Intercooled, versión 8.2 (32) y las matrices de gasto se elaboraron en Microsoft ${ }^{\circledR}$ Excel 2002 (33).

\section{RESULTADOS}

El gasto en salud reproductiva en México en 2003 fue de 2 912,6 millones de dólares estadounidenses. La distribución del gasto entre los agentes de financiamiento se presenta en la figura 1. Si bien $53,5 \%$ del gasto en salud reproductiva fue público, una parte importante $(43,7 \%)$ provino directamente de los hogares.

Como se observa en el cuadro 1, el programa de salud materno-perinatal presentó el mayor gasto $(85,0 \%$ del gasto en salud reproductiva), fundamentalmente por los pagos directos de los hogares. Las funciones de salud materno-perinatal, donde se concentró la mayor parte del gasto, estuvieron relacionadas principalmente con los servicios de asistencia curativa — como los partos y las cesáreas y la atención de complicaciones - tanto durante el embarazo y el puerperio como en el recién nacido (cuadro 2). Se debe subrayar que si bien las complicaciones representaron $30,6 \%$ del gasto total en 
FIGURA 1. Distribución del gasto en salud reproductiva entre agentes de financiamiento, 2003

Gobierno: 13,3\%

Seguridad Social: $40,2 \%$

Pagos directos de los hogares: $43,7 \%$

Seguros privados: $2,5 \%$

ONG ${ }^{\mathrm{a}}: 0,3 \%$

a ONG: Organizaciones no gubernamentales.
Sector público: $53,5 \%$

Sector privado: $46,5 \%$
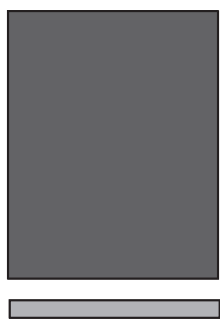
ales. este programa, los agentes de la seguridad social le destinaron $64,8 \%$ de su gasto en salud materno-perinatal (cuadro 2). El gasto en los servicios de prevención y salud pública (como las consultas prenatales) representó 14,0\% del gasto en salud materno-perinatal, aunque estos servicios representaron $42,2 \%$ del gasto de las empresas priva- das de seguro y las ONG en este programa (cuadro 2).

Los servicios destinados a la planificación familiar representaron 5,9\% del gasto total en salud reproductiva y fueron financiados mayoritariamente por los agentes del sector público (cuadro 1). La función de salud a la que los agentes de financiamiento destinaron mayores recursos dentro de este programa fue la prevención (consultas y asesorías de planificación familiar) (cuadro 2). La adquisición de métodos anticonceptivos (englobada en productos médicos dispensados a pacientes ambulatorios) representó 34,7\% del gasto en planificación familiar. El patrón de distribución del gasto de los agentes de financiamiento por funciones no fue homogéneo: mientras los agentes privados dedicaron la mayor proporción $(97,0 \%)$ de su gasto a la adquisición de productos médicos, las entidades del gobierno lo hicieron en los servicios de prevención. La seguridad social, en cambio, equilibró su gasto de planificación familiar entre los servicios de asistencia curativa, los productos médicos y los servicios de prevención (cuadro 2).

En general, 5,7\% del gasto en salud reproductiva se concentró en el programa de cáncer cervicouterino y 2,2\% en el de cáncer de mama (cuadro 1). En ambos casos, el gasto principal se dedicó al tratamiento de esas enfermedades en los servicios de asistencia curativa (cuadro 2). En el caso del cáncer cervicouterino, la segunda función con mayor gasto fueron los servicios auxiliares de atención de la salud (debido fundamentalmente al gasto en las pruebas citológicas), mientras que para el cáncer de mama, la segunda función con mayor gasto fue la relacionada con

CUADRO 1. Gasto estimado en salud reproductiva según los agentes de financiamiento, por programa. México, 2003a

\begin{tabular}{|c|c|c|c|c|c|}
\hline \multirow[b]{2}{*}{ Programa } & \multirow[b]{2}{*}{ Total $^{b}$} & \multicolumn{2}{|c|}{ Sector público } & \multicolumn{2}{|c|}{ Sector privado } \\
\hline & & Gobiernoc & $\begin{array}{l}\text { Seguridad } \\
\text { social }^{c}\end{array}$ & $\begin{array}{c}\text { Pagos } \\
\text { directos de } \\
\text { los hogares }{ }^{c}\end{array}$ & $\begin{array}{c}\text { Empresas } \\
\text { privadas de } \\
\text { seguro y ONG }{ }^{c, d}\end{array}$ \\
\hline 1. Salud materno-perinatal & $2475,7(85,0)$ & $252,8(10,2)$ & $971,2(39,3)$ & $1189,1(48,0)$ & $62,6(2,5)$ \\
\hline 2. Planificación familiar & $171,3(5,9)$ & $81,9(47,8)$ & $53,5(31,2)$ & $31,6(18,5)$ & $4,3(2,5)$ \\
\hline 3. Cáncer cervicouterino & $165,1(5,7)$ & $26,5(16,1)$ & $86,3(52,3)$ & $50,4(30,5)$ & $1,9(1,1)$ \\
\hline 4. Cáncer de mama & $63,7(2,2)$ & $10,4(16,3)$ & $40,1(63,0)$ & $0,6(0,9)$ & $12,6(19,8)$ \\
\hline \multicolumn{6}{|l|}{ 5. Rectoría y administración de la salud } \\
\hline y los seguros médicos & $26,5(0,9)$ & $6,3(23,8)$ & $20,2(76,2)$ & $\mathrm{NI}$ & $\mathrm{NI}$ \\
\hline \multicolumn{6}{|l|}{ 6. Formación de capital, formación del personal } \\
\hline sanitario e investigación y desarrollo & $10,3(0,3)$ & $10,3(100)$ & $\mathrm{NI}$ & $\mathrm{NI}$ & $\mathrm{NI}$ \\
\hline Total & 2912,6 & $388,2(13,3)$ & $1171,3(40,2)$ & $1271,7(43,7)$ & $81,4(2,8)$ \\
\hline
\end{tabular}

a En millones de dólares estadounidenses. Tipo de cambio: 1 dólar estadounidense = 10,78 pesos mexicanos.

${ }^{b}$ Entre paréntesis se ofrecen los porcentaje con respecto al gasto total en salud reproductiva.

c Entre paréntesis se ofrecen los porcentajes con respecto al gasto total en el programa.

d ONG: organización no gubernamental.

${ }^{\text {e }} \mathrm{Nl}$ : información desconocida. 
CUADRO 2. Gasto estimado en salud reproductiva según los cuatro principales programas de salud reproductiva y los agentes de financiamiento, desglosado por función de salud. México, 2003 ${ }^{\mathrm{a}}$

\begin{tabular}{|c|c|c|c|c|c|}
\hline \multirow[b]{2}{*}{ Programa/función } & \multirow[b]{2}{*}{ Total } & \multicolumn{2}{|c|}{ Sector público } & \multicolumn{2}{|c|}{ Sector privado } \\
\hline & & Gobierno & $\begin{array}{c}\text { Seguridad } \\
\text { social }\end{array}$ & $\begin{array}{c}\text { Pagos } \\
\text { directos de } \\
\text { los hogares }\end{array}$ & $\begin{array}{c}\text { Empresas } \\
\text { privadas de } \\
\text { seguro y ONG }\end{array}$ \\
\hline \multirow{4}{*}{$\begin{array}{l}\text { 1. Salud materno-perinatal } \\
\text { 1.1 Servicios de asistencia curativa } \\
\text { Partos y cesáreas } \\
\text { Complicaciones en el embarazo, parto, } \\
\text { puerperio y recién nacido }\end{array}$} & 2475,7 & 252,8 & 971,2 & 1189,1 & 62,6 \\
\hline & 2010,8 & 212,9 & 812,9 & 950,1 & 34,9 \\
\hline & $1191,8(48,2)$ & $100,9(39,9)$ & $137,4(14,1)$ & $918,7(77,3)$ & $34,8(55,6)$ \\
\hline & $757,7(30,6)$ & $97,1(38,4)$ & $629,2(64,8)$ & $31,4(2,6)$ & $N I^{d}$ \\
\hline Otros ${ }^{c}$ & $61,3(2,4)$ & $14,9(5,9)$ & $46,3(4,8)$ & $0,0(0,0)$ & $0,1(0,2)$ \\
\hline 1.2 Servicios auxiliares de atención de la salud & $51,2(2,1)$ & $\mathrm{NI}$ & $\mathrm{NI}$ & $49,9(4,2)$ & $1,3(2,0)$ \\
\hline 1.3 Productos médicos dispensados a pacientes & & & & & \\
\hline ambulatorios & $67,6(2,7)$ & $1,0(0,4)$ & $\mathrm{NI}$ & $66,6(5,6)$ & $\mathrm{NI}$ \\
\hline 1.4 Servicios de prevención y de salud pública & $346,1(14,0)$ & $38,9(15,4)$ & $158,3(16,3)$ & $122,5(10,3)$ & $26,4(42,2)$ \\
\hline 2. Planificación familiar & 171,3 & 81,9 & 53,5 & 31,6 & 4,3 \\
\hline 2.1 Servicios de asistencia curativa & $34,0(19,9)$ & $14,5(17,7)$ & $19,5(36,5)$ & $\mathrm{NI}$ & $0,0(0,7)$ \\
\hline 2.2 Productos médicos dispensados a pacientes & & & & & \\
\hline ambulatorios & $59,4(34,7)$ & $9,3(11,4)$ & $14,3(26,7)$ & $31,6(100,0)$ & $4,2(97,0)$ \\
\hline 2.3 Servicios de prevención y de salud pública & $77,9(45,4)$ & $58,1(70,9)$ & $19,7(36,8)$ & $\mathrm{NI}$ & $0,1(2,3)$ \\
\hline 3. Cáncer cervicouterino & 165,1 & 26,5 & 86,3 & 50,4 & 1,9 \\
\hline 3.1 Servicios de asistencia curativa & $145,2(87,9)$ & $6,8(25,7)$ & $86,3(100,0)$ & $50,4(100,0)$ & $1,7(91,5)$ \\
\hline 3.2 Servicios auxiliares de atención de la salud & $15,2(9,2)$ & $15,0(56,6)$ & $\mathrm{NI}$ & $\mathrm{NI}$ & $0,2(8,5)$ \\
\hline 3.3 Servicios de prevención y de salud pública & $4,7(2,9)$ & $4,7(17,7)$ & $\mathrm{NI}$ & $\mathrm{NI}$ & $\mathrm{NI}$ \\
\hline 4. Cáncer de mama & 63,7 & 10,4 & 40,1 & 0,6 & 12,6 \\
\hline 4.1 Servicios de asistencia curativa & $58,0(91,1)$ & $5,0(48,1)$ & $40,1(100,0)$ & $0,6(100,0)$ & $12,3(97,8)$ \\
\hline 4.3 Servicios auxiliares de atención de la salud & $0,4(0,6)$ & $0,1(1,0)$ & $\mathrm{NI}$ & $\mathrm{NI}$ & $0,3(2,2)$ \\
\hline 4.2 Servicios de prevención y de salud pública & $5,3(8,3)$ & $5,3(51,0)$ & $\mathrm{NI}$ & $\mathrm{NI}$ & $\mathrm{NI}$ \\
\hline
\end{tabular}

los servicios de prevención $(8,4 \%)$, ambas financiadas casi exclusivamente por el estado (cuadro 2). La seguridad social fue el principal agente de financiamiento, tanto para el diagnóstico y tratamiento del cáncer cervicouterino $(52,3 \%)$ como del de mama $(63,0 \%)$ (cuadro 1).

El gasto relacionado con la rectoría y administración de los servicios de salud y con los seguros médicos representó $0,9 \%$ del gasto total en salud reproductiva, mientras que a otras funciones relacionadas con la salud - como la formación bruta de capital y de personal sanitario, así como las actividades de investigación y desarrollocorrespondió $0,3 \%$ de ese gasto. El financiamiento de ambas actividades estuvo totalmente a cargo de agentes del sector público (cuadro 1).
En cuanto a los proveedores, 72,9\% del gasto en salud reproductiva correspondió a los hospitales y $21,2 \%$ a los proveedores de atención ambulatoria (cuadro 3). Mientras el gasto en salud materno-perinatal se concentró en los hospitales (para la atención de los partos, las cesáreas y las complicaciones), el gasto en planificación familiar se concentró en los proveedores de atención ambulatoria, donde se brindaron las consultas y asesorías de planificación familiar (43,5\%). En general, 74,9\% del gasto en cáncer cervicouterino se destinó a pagar a los proveedores de atención ambulatoria (quienes brindaron consultas especializadas en este tipo de cáncer y realizaron pruebas citológicas y biopsias, entre otras tareas); $82,1 \%$ del gasto en cáncer de mama correspondió a los hospitales (cuadro 3).
Al igual que a nivel nacional, el gasto público de los estados en salud reproductiva se concentró en el programa de salud materno-perinatal y en el de planificación familiar (77,5\% y $10,5 \%$, en promedio, respectivamente). El programa de cáncer cervicouterino representó $8,1 \%$ de ese gasto y el de cáncer de mama 3,1\%, mientras la rectoría y administración de la salud y las funciones de formación de capital, de personal sanitario y de investigación y desarrollo, en conjunto, representaron en promedio $0,8 \%$ del gasto de los estados (figura 2).

El promedio del gasto público por beneficiaria en salud reproductiva a nivel estatal fue de 680,03 dólares estadounidenses. En los estados se observó una relación directa entre el gasto público en salud reproductiva 
CUADRO 3. Gasto estimado en salud reproductiva según los proveedores, por programa de salud. México, 2003a

\begin{tabular}{|c|c|c|c|c|c|c|}
\hline Programa & Total & Hospitales & $\begin{array}{l}\text { Proveedores } \\
\text { de atención } \\
\text { ambulatoria }\end{array}$ & Farmacias & $\begin{array}{l}\text { Programas } \\
\text { de salud } \\
\text { pública }\end{array}$ & $\begin{array}{l}\text { Administración } \\
\text { general de } \\
\text { la salud }\end{array}$ \\
\hline Salud materno-perinatal & 2475,7 & $1993,4(80,5)$ & $412,0(16,7)$ & $67,0(2,7)$ & $3,3(0,1)$ & $\mathrm{NI}^{\mathrm{b}}$ \\
\hline Planificación familiar & 171,3 & $36,9(21,5)$ & $74,5(43,5)$ & $59,4(34,7)$ & $0,5(0,3)$ & $\mathrm{NI}$ \\
\hline Cáncer cervicouterino & 165,1 & $39,5(23,9)$ & $123,6(74,9)$ & $\mathrm{NI}$ & $2,0(1,2)$ & $\mathrm{NI}$ \\
\hline Cáncer de mama & 63,7 & $52,3(82,1)$ & $8,7(13,7)$ & $\mathrm{NI}$ & $2,7(4,2)$ & $\mathrm{NI}$ \\
\hline \multicolumn{7}{|l|}{ Rectoría y administración } \\
\hline de la salud y los seguros médicos & 26,5 & $\mathrm{NI}$ & $\mathrm{NI}$ & $\mathrm{NI}$ & $\mathrm{NI}$ & $26,5(100)$ \\
\hline \multicolumn{7}{|l|}{ Formación de capital y de personal } \\
\hline sanitario e investigación y desarrollo & 10,3 & $\mathrm{NI}$ & $\mathrm{NI}$ & $\mathrm{NI}$ & $\mathrm{NI}$ & $10,3(100)$ \\
\hline Total & 2912,6 & $2122,1(72,9)$ & $618,8(21,2)$ & $126,4(4,3)$ & $8,5(0,3)$ & $36,8(1,3)$ \\
\hline
\end{tabular}

a En millones de dólares estadounidenses. Tipo de cambio: 1 dólar estadounidense $=10,78$ pesos mexicanos. En paréntesis se ofrecen los porcentajes con respecto al gasto total en el programa.

b NI: información no existente.

por beneficiaria y el PIB anual per cápita y entre el gasto público en salud per cápita y el gasto público en salud reproductiva por beneficiaria (figura 3). Según estos resultados, los estados con mayor PIB tuvieron mayor gasto público en salud reproductiva por beneficiaria $(r=0,75, P<0,001)$ y los estados donde el gasto público per cápita en salud fue alto, también fue mayor el gasto público en salud reproductiva por beneficiaria $(r=0,80, P<$ $0,001)$. Vale la pena resaltar los casos de Baja California Sur y Yucatán, ya que tuvieron un mayor gasto público en salud reproductiva por beneficiaria que otros estados con niveles similares de PIB y de gasto público en salud per cápita. Por el contrario, el estado de Guerrero tuvo el menor gasto público en salud reproductiva por beneficiaria, a pesar de que su PIB anual y su gasto público en salud per cápita fueron similares a los de otros estados - como Tlaxcala- que tuvieron un mayor gasto en salud reproductiva.
FIGURA 2. Distribución del gasto público promedio en salud reproductiva a nivel estatal. México, 2003

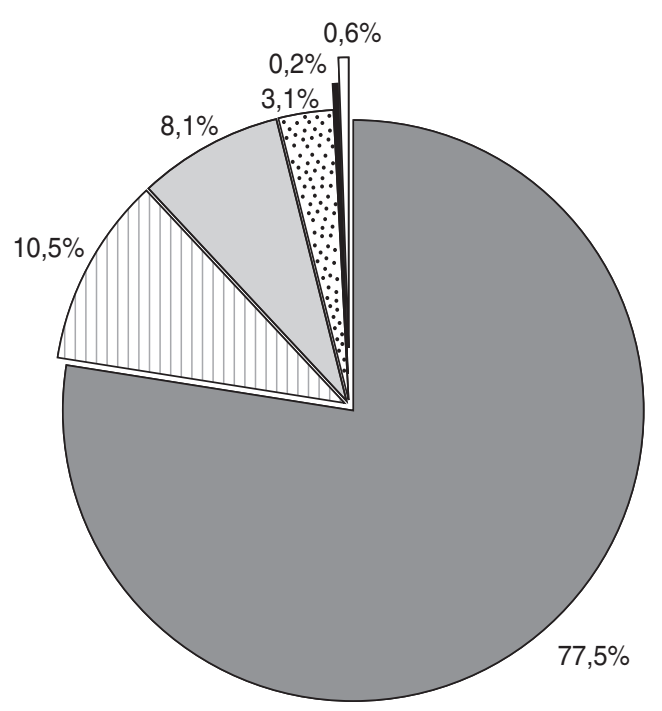

Salud materno-perinatal

Planificación familiar

Cáncer cervicouterino

Cáncer de mama

Rectoría y administración

Otros

\section{DISCUSIÓN}

La importancia de que la población disfrute de una buena salud reproductiva es vital para poder alcanzar los Objetivos de Desarrollo del Milenio, ya que la pobreza y la mala salud reproductiva están íntimamente ligadas (35). Las poblaciones más pobres y marginadas presentan por lo general los peores indicadores de salud reproductiva (mayor número de hijos de los deseados o planeados, mayores complicaciones durante el parto, el aborto y el puerperio, elevada morbimortalidad materna y perinatal, alta prevalencia de infecciones de transmisión sexual e índices elevados de embarazo precoz, abandono escolar y orfandad prematura). Todo esto contribuye a perpetuar el círculo vicioso de la pobreza. A pesar de ello, la competencia por recursos en salud, las presiones ideológicas y la existencia de diversos padecimientos emergentes han reducido los esfuerzos dirigidos a mejorar la salud reproductiva.

Conocer el gasto que un país realiza en salud reproductiva es indispensable para velar por el cumplimiento de los compromisos y las acciones a nivel nacional e internacional; identificar los programas, las actividades, las funciones o los grupos de la población que se encuentran subfinanciados; y reorientar la asignación del presupuesto de acuerdo con las necesidades, las 
FIGURA 3. Gasto público en salud reproductiva por beneficiaria y su relación con el gasto público per cápita en salud y el producto interno bruto per cápita. México, 2003a

A. Gasto público en salud reproductiva por beneficiaria según el producto interno bruto per cápita de los estados

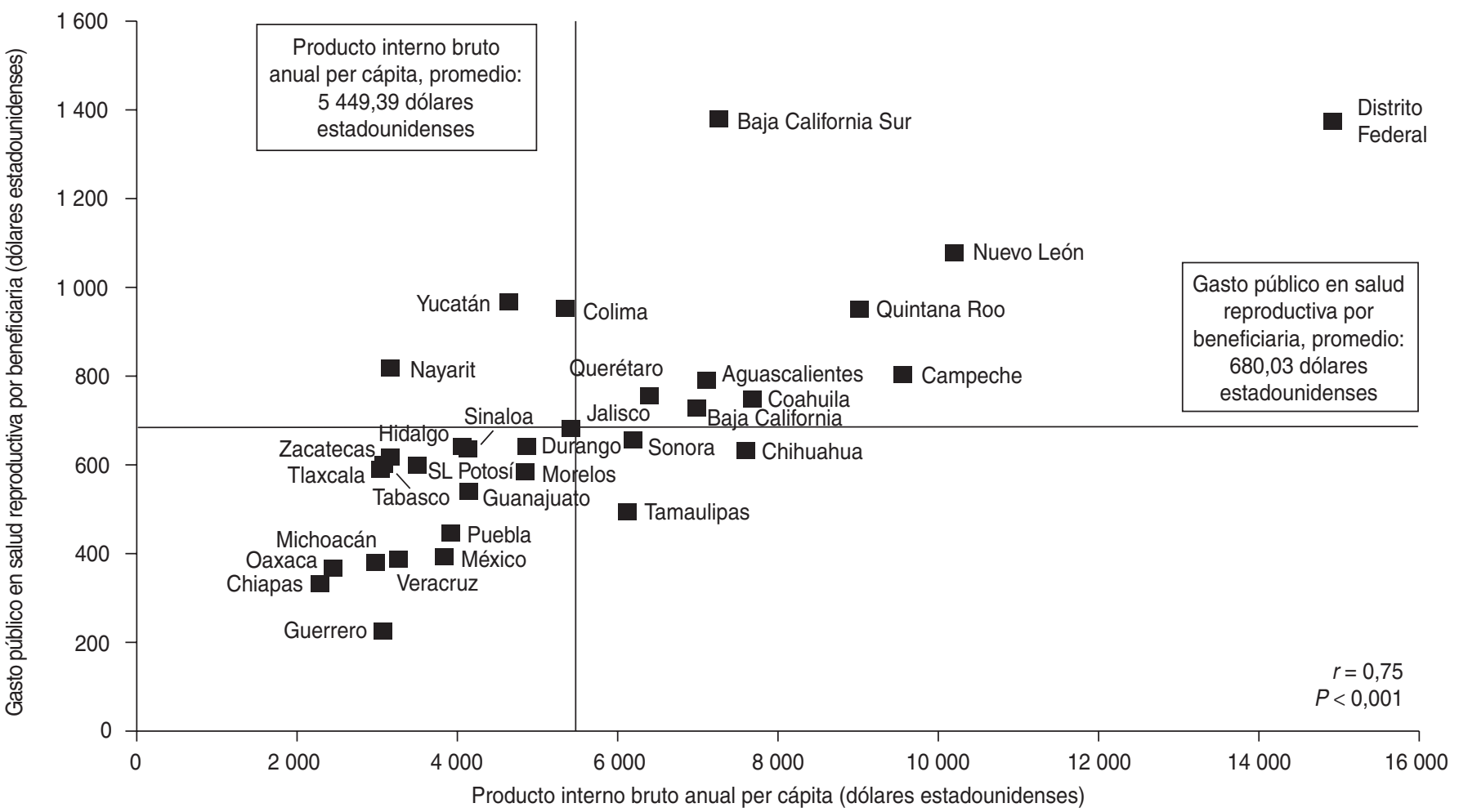

B. Gasto público en salud reproductiva por beneficiaria según el gasto público en salud per cápita de los estados

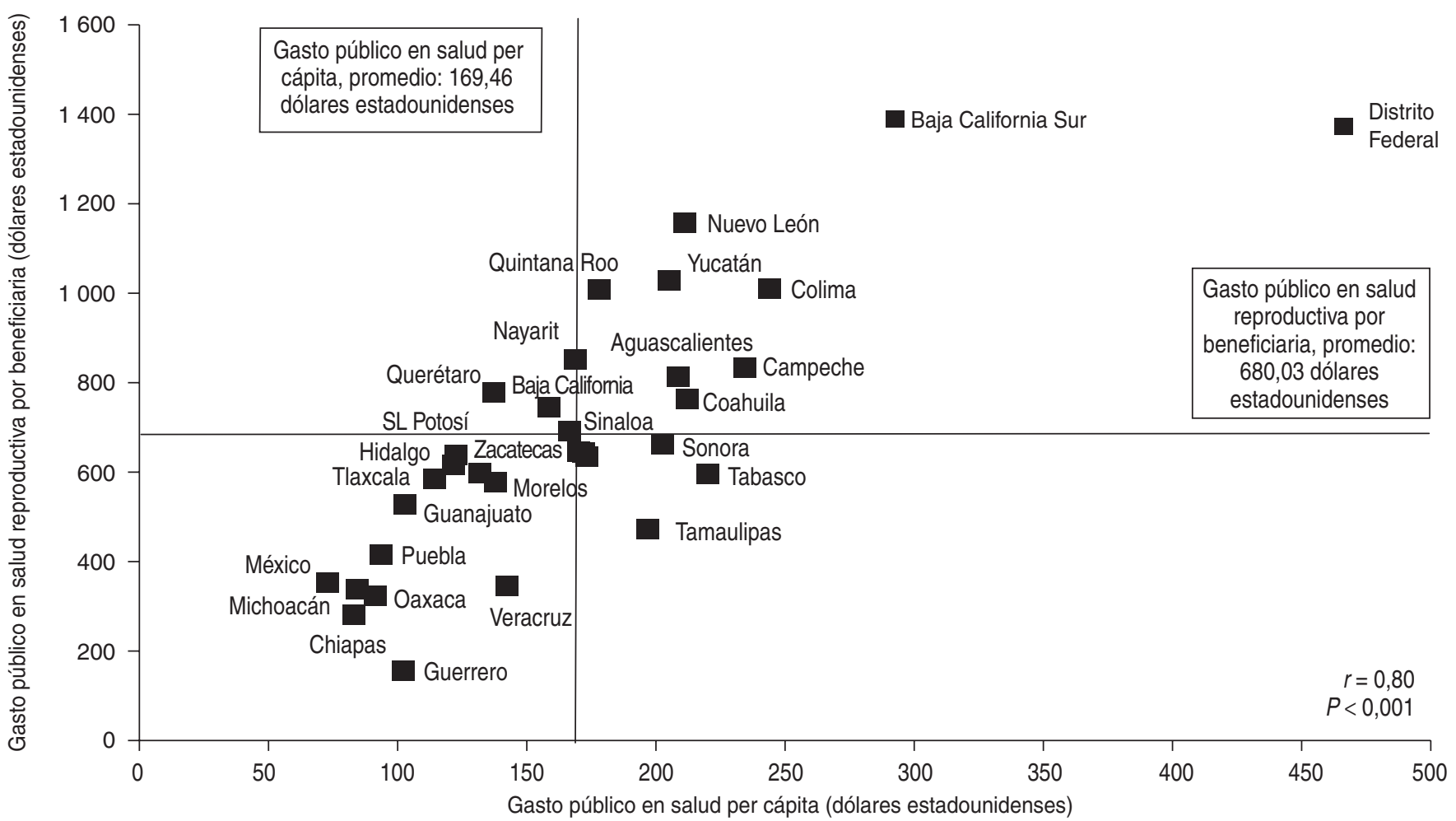

Fuentes: Ref. 10 y 34.

a Gastos expresados en dólares estadounidenses al cambio promedio de 2003. Tipo de cambio: 1 dólar estadounidense = 10,78 pesos mexicanos. 
prioridades e intervenciones que han demostrado tener una mayor efectividad en función del costo. Por otro lado, conocer la estructura del gasto en salud reproductiva permite realizar comparaciones entre instituciones, localidades y países en diferentes contextos, como un insumo adicional para la toma de decisiones y una forma de aumentar la transparencia y la rendición de cuentas.

Este trabajo contribuye a conocer el panorama financiero de la salud reproductiva en México, mediante una adaptación de la metodología de cuentas en salud, y brinda información para la evaluación del desempeño y el seguimiento del efecto de las políticas y las reformas relacionadas con la asignación de recursos.

Los resultados obtenidos muestran que, al igual que en el gasto total en salud, la salud reproductiva se financió en gran medida mediante el pago directo de los hogares. El gasto de la seguridad social representó un porcentaje del gasto en salud reproductiva mayor que del gasto en salud $(40,2 \%$ frente a $31,0 \%$, respectivamente). Si bien estas cifras parecen indicar una mejor distribución de la carga financiera de la salud reproductiva frente al gasto total en salud, la fuerte contribución de los pagos directos de los hogares es un importante reto para el sistema de salud mexicano. La puesta en marcha del Seguro Popular, cuyo catálogo de intervenciones abarca la mayoría de las actividades de salud reproductiva estudiadas, busca revertir esta situación y proteger a las familias de incurrir en gastos en salud que podrían afectar más aun a su situación económica. Análisis posteriores de contabilidad en salud deben evaluar si la implantación de este nuevo seguro permitirá reducir los pagos directos de los hogares en esta área, ya que estos afectan más a las mujeres de bajos ingresos, las indígenas, las adolescentes y a otros grupos prioritarios de la población. Es precisamente con respecto a estos grupos poblacionales más desfavorecidos que se plantean los mayores retos y se requiere fortalecer los programas de salud reproductiva.
La metodología utilizada permite, además, especificar la distribución del gasto en los principales programas y las diferentes funciones en salud reproductiva. Este punto es de especial importancia dado que el Gobierno mexicano ha establecido compromisos para alcanzar los Objetivos de Desarrollo del Milenio relacionados con la reducción de la mortalidad materna y en niños menores de cinco años (36), lo que ha llevado a establecer explícitamente metas nacionales en el Plan Nacional de Salud 2001-2006 (37). Entre estas metas nacionales se encuentran garantizar condiciones de equidad en el comienzo de la vida mediante actividades que garanticen un embarazo planeado y deseado, la atención prenatal adecuada y el nacimiento con un mínimo de riesgos; aumentar la cobertura de detección temprana de cáncer cervicouterino y disminuir la tasa de mortalidad por esta enfermedad; reducir la tasa general de fecundidad a 2,1 hijos por mujer; y detener el aumento en la tasa de mortalidad por cáncer de mama.

Al igual que en otros países (38-40), en México se dedica la mayor proporción del gasto en salud reproductiva al programa de salud materno-perinatal. A pesar de que el rubro con mayor gasto fue el de partos y cesáreas, llama la atención el alto monto destinado a la atención de complicaciones en el embarazo, el parto, el puerperio y el recién nacido, que asciende a más del doble del empleado en acciones preventivas en salud materno-perinatal e incluso supera varias veces el destinado a los otros programas de salud reproductiva. Si bien el mayor tiempo de estancia hospitalaria es una explicación para este hecho, los resultados parecen indicar que esta situación responde al uso ineficiente de los recursos dedicados a la atención obstétrica, lo que deberá analizarse a la luz de las recomendaciones de la OMS (41). Se requiere, por tanto, realizar un estudio en profundidad para esclarecer las causas de estas complicaciones, la comparación entre el número de complicaciones y el número de partos por institución y su relación con la cobertura eficaz del control prenatal, tanto en el ámbito nacional como estatal.
Otro punto a resaltar es la forma en que los agentes de financiamiento distribuyen el gasto entre las funciones de salud. Mientras los pagos directos de los hogares se dirigen a la atención de los partos y las cesáreas y a la atención prenatal, el gasto de la seguridad social se dedica al tratamiento de complicaciones durante el embarazo, el parto y el puerperio y a atender los casos de cáncer cervicouterino y de mama. Se debe subrayar que el monto del gasto público destinado por la seguridad social a atender las complicaciones es 4,6 veces el monto gastado por ese mismo agente en la atención de partos y cesáreas.

Con relación al gasto realizado por los proveedores de salud, algunos elementos pueden ayudar a mejorar el uso de los recursos. La concentración del gasto en actividades hospitalarias en detrimento de las actividades preventivas - como las consultas de control prenatal, de recién nacido sano y de puerperio y las asesorías de planificación familiar- podría ser, en parte, el reflejo de los problemas anteriormente señalados. No se puede descartar, sin embargo, que otros factores relacionados con la oferta o la demanda de los servicios de salud puedan estar influyendo en esta forma de distribución del gasto. Por ejemplo, el sistema de referencia y contrarreferencia en las instituciones pertenecientes a la Secretaría de Salud de un estado y las preferencias de la población condujeron a la saturación del servicio de urgencias en un hospital, cuando en realidad muchos de esos partos se hubieran podido atender en centros de salud (42). Es necesario, por tanto, profundizar en el análisis de estos factores y valorar si realmente se está cumpliendo el objetivo de fortalecer la capacidad resolutiva del nivel de atención primaria propuesto en el Plan Nacional de Salud (37).

El gasto público realizado en los diferentes estados presentó una gran heterogeneidad. La asociación directa observada entre el gasto público por beneficiaria, el gasto público en salud y el PIB anual per cápita de los estados indica que el proceso de descentralización no ha logrado aún revertir la ten- 
dencia histórica en la distribución del gasto público y que todavía no se asignan los recursos públicos de acuerdo con las necesidades locales de salud. Asimismo, llama la atención que estados con el mismo nivel de PIB y gasto público en salud per cápita tengan tan diferentes niveles de gasto en salud reproductiva. Si bien esto podría ser producto de diferentes necesidades en salud, es necesario profundizar en este análisis para establecer cuál podría ser la asignación óptima de recursos en cada estado, dadas sus características, necesidad y disponibilidad financiera. Se debe evaluar si también hay asociación entre el gasto en salud reproductiva y otros indicadores de salud, como la tasa de mortalidad materna, por cáncer cervicouterino y de mama y la tasa de fecundidad. Un análisis reciente de la relación entre la cobertura y el financiamiento del programa de planificación familiar encontró que, si bien el gasto público está asociado negativamente con la proporción de mujeres en edad fértil (indicador de demanda potencial de servicios de planificación familiar) en un estado, no se observó una asociación significativa con otros indicadores compuestos de necesidades de salud en planificación familiar.

A pesar del esfuerzo realizado en la construcción de las cuentas en salud reproductiva, se presentaron limitaciones en su estimación y construcción. Debido a que la ENIGH no tiene representatividad estatal, no se pudo estimar el gasto privado a ese nivel y la información desagregada de los gastos correspondientes a los diferentes programas de salud no estuvo disponible en todos los estados. Asimismo, las limitaciones de las encuestas utilizadas solo permitieron calcular parcialmente el gasto en displasias, cáncer cervicouterino y de mama, por lo que los pagos directos de los hogares en esos rubros están subestimados. El análisis presentado para cada estado se refiere al gasto por beneficiaria y no al gasto per cápita, medida generalmente utilizada en la comparación del gasto. La gran variabilidad en los datos informados por las diferentes fuentes oficiales con respecto a la población a la que cada institución da cobertura, tal y como se señala en el informe de OECD (43), constituyó un importante reto para la construcción de estos indicadores. Esta heterogeneidad obligó a utilizar distintos métodos de estimación.

Los resultados obtenidos solo representan el panorama financiero de un conjunto de actividades relacionadas con la salud reproductiva. Aun cuando la información presentada expone problemas de ineficiencia en el uso de los recursos, para evaluar el desempeño del sistema de salud es necesario tomar en consideración otros indicadores de salud y se debe analizar la tendencia del gasto.

A pesar de las limitaciones señaladas, este estudio es de gran utilidad y constituye el primer esfuerzo por desglosar la información financiera de las cuentas en salud según los programas específicos existentes en México, tanto a nivel nacional como estatal. En el mundo son muy pocos los estudios que han aplicado este tipo de estudios a la salud reproductiva (38-40).

Estos resultados permiten concluir que el gasto en salud reproductiva representó 0,5\% del PIB mexicano en 2003 y poco más de $8 \%$ del gasto en salud y es mayor en agentes públicos $(53,5 \%)$ que en privados $(46,5 \%)$, lo contrario de lo que ocurre con el gasto total en salud. La distribución del gasto en salud reproductiva por beneficiaria a nivel estatal estuvo directamente asociada con la distribución del PIB y el gasto en salud per cápita.

En México se han logrado grandes avances en la recopilación y procesamiento de la información financiera, no solo de salud en general, sino también en el área específica de la salud reproductiva. Sin embargo, todavía quedan retos para el futuro. Se recomienda ampliar la definición de salud reproductiva y generar este tipo de información financiera de forma sistemática, institucionalizada y comparable. De esta manera se podrán construir series de tiempo que permitirán entender mejor las tendencias del gasto y su relación con los indicadores de salud. Además, esto permitirá evaluar el desempeño del sistema de salud y el impacto de medidas - como la creación del Seguro Popular- y favorecerá la rendición de cuentas en un contexto de mayor democracia.

Invertir en la salud reproductiva ayudará a reducir el crecimiento demográfico, optimizar los recursos educativos y laborales y, sobre todo, permitirá a las personas y a las parejas ejercer más plenamente sus derechos y aspiraciones reproductivas. Fortalecer las acciones y los programas de salud reproductiva en México a partir de una asignación del gasto basada en la evidencia y enfocada a las poblaciones más desfavorecidas es un imperativo ético, de derechos humanos y de desarrollo.

Agradecimientos. Este artículo forma parte del proyecto Cuentas Nacionales en Salud Reproductiva y Equidad de Género, que fue financiado por el Centro Nacional de Equidad de Género y Salud Reproductiva de la Secretaría de Salud de México.

\section{REFERENCIAS}

1. Merino-Juárez MF, Sesma-Vásquez, S. Gasto público en salud 1999-2000. Síntesis ejecutiva [publicación en Internet]. Secretaría de Salud. 2001. Hallado en: http://www.salud.gob. $\mathrm{mx} /$ apps/htdocs/sicuentas/publicaciones/ gasto_p.pdf. Acceso el 22 de septiembre de 2006.
2. Zurita $B$, Hernández $P$, Ramírez TJ, Méndez O, Gamble A, Cruz M. Cuentas Nacionales de Salud. México, D.F.: Fundación Mexicana para la Salud; 1998.

3. Fundar, Centro de Análisis e Investigación. Muerte materna y presupuesto público. México, D.F.: Fundar; 2004.
4. Estados Unidos Mexicanos, Secretaría de Salud. México salud 2004: información para la rendición de cuentas. 2. ${ }^{a}$ ed [publicación en Internet]. México, D.F.: Secretaría de Salud; 2005. Hallado en: http://evaluacion.salud. gob.mx/saludmex2004/sm2004.pdf. Acceso el 22 de septiembre de 2006. 
5. Estados Unidos Mexicanos, Secretaría de Salud. México salud 2003: información para la rendición de cuentas. 2. ${ }^{\text {a }}$ ed [publicación en Internet]. México, D.F.: Secretaría de Salud; 2004. Hallado en: http://www.salud.gob. $\mathrm{mx} /$ unidades/evaluacion/saludmex2003/ sm2003.pdf. Acceso el 22 de septiembre de 2006.

6. Instituto Mexicano del Seguro Social. Memoria estadística 2003 [CD Rom]. Méxio, D.F.: Instituto Mexicano del Seguro Social; 2004

7. Instituto Nacional de Estadística, Geografía e Informática. Distribución porcentual de la población usuaria de servicios de salud según tipo de institución para cada sexo y grupo de edad, 2000 [sitio en Internet]. México, D.F.: INEGI. Hallado en: http://www.inegi.gob. $\mathrm{mx} / \mathrm{est} / \mathrm{contenidos/espanol/rutinas/ept.asp}$

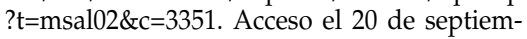
bre de 2006 .

8. Instituto de Servicio y Seguridad Social de los Trabajadores del Estado. Anuario estadístico 2003 [CD Rom]. México, D.F.: ISSSTE; 2004.

9. Estados Unidos Mexicanos, Secretaría de Salud. Matrices OCDE 1999-2003 [sitio en internet]. México, D.F.: SICUENTAS; 2005. Hallado en: http://www.salud.gob.mx/apps/ htdocs/sicuentas/publicaciones/Matrices9903_sp.xls. Acceso el 22 de septiembre de 2006.

10. Estados Unidos Mexicanos, Secretaría de Salud. Gasto público en salud. México 1999-2003 [sitio en Internet]. México, D.F.: SICUENTAS. Hallado en: http://www.salud.gob.mx/apps / htdocs/sicuentas/publicaciones/GastoPublico enSalud_sp.xls. Acceso el 22 de septiembre de 2006.

11. Instituto Nacional de Estadística, Geografía e Informática. Producto interno bruto nominal trimestral según gran división [sitio en Internet]. México, D.F.: INEGI. Hallado en: http:/ / www.inegi.gob.mx/est/contenidos/espanol/ rutinas $/$ ept.asp?t=agr02\&c $=469$. Acceso el 22 de septiembre de 2006.

12. Ávila L, Cahuana L, Pérez R. Cuentas nacionales en salud reproductiva y equidad de género. México, D.F.: Secretaría de Salud, Instituto Nacional de Salud Pública; 2005.

13. World Bank, World Health Organization, United States Agency for International Development. Guide to producing national health accounts with special applications for lowincome and middle-income countries. Vancouver (Canada): World Health Organization; 2003.

14. Merino-Juárez MF, Alarcón-Gómez M, LozanoAscencio R. Manual del Sistema de Cuentas Nacionales y Estatales de Salud. México, D.F.: Dirección General de Información en Salud; 2004.

15. World Health Organization. Reproductive health [sitio en Internet]. Geneva: WHO. Hallado en: http://www.who.int/topics / reproductive_health/en/. Acceso el 20 de septiembre de 2005.

16. Secretaría de Salud. Programa de acción: salud reproductiva. México, D.F.: Secretaría de Salud; 2001.

17. Isasola JA. Sistemas de información de respuestas nacionales contra el SIDA: indicadores financieros. Flujos de financiamiento y gasto en VIH/SIDA. Cuentas Nacionales en VIH/SIDA. México, D.F.: Fundación Mexicana para la Salud; 2002.
18. Organización Panamericana de la Salud. Clasificación estadística internacional de enfermedades y problemas relacionados con la salud. $10^{\mathrm{a}}$ ed. Washington, D.C.: OPS; 1995.

19. Hernández-Peña $P$, Lazcano-Ponce EC, Alonso-de Ruiz P, Cruz-Valdez A, MenesesGonzález F, Hernández-Ávila M. Análisis de costo beneficio del Programa de Detección Oportuna del Cáncer Cervicouterino. Salud Pub Mex. 1997;39:379-87.

20. Gómez-Jáuregui J. Costos y calidad de la prueba de detección oportuna del cáncer cervicouterino en una clínica pública y en una organización no gubernamental. Salud Pub Mex. 2001;43:279-88.

21. Ávila-Figueroa C, Gómez-Fraga S, Herrera BE, Sousa FA, Lozano AR. Estimación de los costos de producción de servicios clínicos para la prevención, diagnóstico y tratamiento médico. México, D.F.: Secretaría de Salud; 2002.

22. Cahuana-Hurtado L, Sosa-Rubí S, Bertozzi S. Costo de la atención materno infantil en el estado de Morelos, México. Salud Pub Mex. 2004;46:316-25.

23. Legagneur D. Estimación de costos por caso hospitalario en el tratamiento de cáncer cervicouterino en el Hospital Parres en Cuernavaca, Morelos, durante el período enero 2000-diciembre 2002 [tesis de maestría]. Cuernavaca (México): Instituto Nacional de Salud Pública; 2003.

24. Instituto Nacional de Estadística, Geografía e Informática. Encuesta nacional de ingreso y gasto de los hogares. 2002 [CD-ROM]. Aguascalientes (México): INEGI; 2003.

25. Instituto Nacional de Estadística, Geografía e Informática. Encuesta nacional de ingreso y gasto de los hogares. 2004 [CD-ROM]. Aguascalientes (México): INEGI; 2005.

26. Estados Unidos Mexicanos, Secretaría de Salud. Encuesta nacional de salud reproductiva. 2003 [CD-ROM]. México, D.F.: Secretaría de Salud; 2004

27. World Health Organization. Mother-baby package costing spreadsheet. Versión 1.01. [CD-ROM]. Geneva: WHO; 1999.

28. Estados Unidos Mexicanos, Secretaría de Salud. Resolución por la que se modifica la Norma Oficial Mexicana NOM-005-SSA21993. De los servicios de planificación familiar. Diario Oficial de la Federación (México) 200421 de enero.

29. Estados Unidos Mexicanos, Secretaría de Salud. Norma Oficial Mexicana NOM-014SSA2-1994. Para la prevención, detección, diagnóstico, tratamiento, control y vigilancia epidemiológica del cáncer cervicouterino. Diario Oficial de la Federación (México) 1998 6 de marzo.

30. Estados Unidos Mexicanos, Secretaría de Salud. Norma Oficial Mexicana NOM-041SSA2-2002. Para la prevención, diagnóstico, tratamiento, control y vigilancia epidemiológica del cáncer de mama. Diario Oficial de la Federación (México) 200317 de septiembre.

31. Banco de México. Tipos de cambio. Información mensual. Tipo de cambio promedio del período [sitio de Internet]. México, D.F.: BANXICO. Hallado en: http:/ / www.banxico.gob. $\mathrm{mx} /$ SieInternet/consultarDirectorioInternet Action. do? accion $=$ consultarCuadro\&id
Cuadro $=$ CF86\&sector $=6 \&$ locale $=$ es. Acceso el 22 de septiembre de 2006.

32. StataCorp LP. Intercooled Stata 8.2 for Windows. College Station, Texas: StataCorp LP; 2004.

33. Microsoft Corporation. Microsoft Excel, 2002. Redmond, Washington: Microsoft Corporation; 2002.

34. Instituto Nacional de Estadística, Geografía e Informática. Sistema de Cuentas Nacionales de México. Banco de Información Económica [sitio en Internet]. México, D.F.: INEGI; 2005. Hallado en: http://dgcnesyp.inegi.gob.mx/ cgi-win/bdieintsi.exe/NIVM100002\#ARBOL. Acceso el 22 de septiembre de 2006.

35. United Nations. The UN Millenium Development Goals [sitio en internet]. United Nations. Hallado en: http://www.un.org/millennium goals/. Acceso el 20 de septiembre de 2006.

36. Gabinete de Desarrollo Humano y Social. Los Objetivos de Desarrollo del Milenio en México: informe de avance 2005 [publicación en Internet]. México, D.F.: Gabinete de Desarrollo Humano y Social; 2005. Hallado en: http:// www.salud.gob.mx/pagina_principal_ 2005/archivos/avance05_inf_comp.pdf. Acceso el 22 de septiembre de 2006.

37. Estados Unidos Mexicanos, Secretaría de Salud. Metas del Programa Nacional de Salud 2001-2006. Documento de seguimiento [publicación en Internet]. México, D.F.: Secretaría de Salud; 2006. Hallado en: http://evaluacion. salud.gob.mx/metas_pns/metas_pns.pdf. Acceso el 22 de septiembre de 2006.

38. Rannan-Eliya R, Berman P, Eltigani E, De Silva W, Somanathan A, Sumathiratne V. Expenditures for reproductive health services in Egypt and Sri Lanka. Colombo: Institute of Policy Studies; 2000. (Occasional Paper 13.2000).

39. Sharma S, McGreevey W, Kanjilal B, Hotchkiss DR. Reproductive and child health accounts: an application to Rajasthan. Health Policy Plan. 2002;17:314-21.

40. De S, Dmytraczenko T, Chanfreau C, Kombe G, Fairbank A, Nandakumar AK. Measurement of population expenditures at the subnational level using the NHA framework. Bethesda, Maryland: Abt Associates; 2004.

41. World Health Organization. The world health report 2005: make every mother and child count. Geneva: WHO; 2005

42. Cahuana L, Sosa S, Bertozzi SM, Guijarro MT. Eficiencia en la atención de partos. Publicación conjunta sobre la eficiencia en la atención de partos, impacto socioeconómico del VIH/ SIDA, las cuentas nacionales: valor agregado en Cuba y costo en atención de pacientes con VIH-SIDA en la delegación de Morelos del IMSS. La Habana: Instituto Nacional de Salud Pública de México, Instituto Superior de Ciencias Médicas de Santiago de Cuba; 2003.

43. Organización para la Cooperación y el Desarrollo Económicos. Estudios de la OCDE sobre los sistemas de salud. México. México, D.F.: OCDE; 2005

Manuscrito recibido el 14 de septiembre de 2005. Aceptado para publicación, tras revisión, el 11 de septiembre de 2006. 
ABSTRACT Objectives. To estimate reproductive health expenditures in Mexico during 2003;

analyze how costs were distributed across the main programs, funding entities, and providers of health goods and services; and evaluate the relationship between repro-

\section{Analysis of reproductive health expenditures in Mexico, 2003} ductive health expenditures and economic indicators in different states, using health accounts methods.

Methods. We estimated reproductive health expenditures between January and December 2003, at the national and state level. We used health accounts methods adjusted for the particular characteristics of Mexico on the basis of information from public and private sources. Expenditures were calculated for the four main reproductive health programs (maternal-perinatal health, family planning, cervical and uterine cancer, and breast cancer) according to different funding entities, goods and services providers, and functions of health care, in both the public and private sector. We estimated public expenditures by state per beneficiary, and analyzed how these costs were related with pubic health care expenditures and annual per capita gross domestic product (GDP) for each state.

Results. The reproductive health expenditures in Mexico during the year 2003 were US\$ 2.9126 billion, a figure that represented $0.5 \%$ of the national GDP in 2003 and slightly more than $8 \%$ of the total health care expenditures. Costs were higher for public entities (53.5\%) than for private entities (46.5\%). The maternal-perinatal health program accounted for the highest costs, mainly from deliveries and complications; direct payments from households accounted for nearly $50 \%$ of the total figure. Costs for family planning were accrued mainly in the public sector, and represented $5.9 \%$ of the total expenditure. Of the total spending on reproductive health, $7.9 \%$ was devoted to cervical and uterine cancer and breast cancer programs. Mean public expenditures on reproductive health per beneficiary were US $\$ 680.03$, and differences between states were associated with differences in public health expenditures $(r=0.80 ; P<0.001)$ and per capita GDP $(r=0.75 ; P<0.0001)$.

Conclusions. The health accounts method allowed us to estimate reproductive health expenditures in Mexico in 2003. Enhancing reproductive health actions and programs by basing expenditure assignments on evidence and focusing on leastfavored populations is an ethical, human rights, and developmental imperative.

Key words Health expenditures, health care costs, reproductive health services, Mexico. 